\title{
miRNA-331-3p Affects The Proliferation, Metastasis And Invasion of Osteosarcoma Through SOCS1/JAK2/STAT3
}

\section{Dan Zu}

The Central Hospital Affiliated to Shaoxing University

\section{Qi Dong}

The Central Hospital Affiliated to Shaoxing University

\section{Sunfang Chen}

The Central Hospital Affiliated to Shaoxing University

\section{Yongde Chen}

The Central Hospital Affiliated to Shaoxing University

Jun Yao

The Central Hospital Affiliated to Shaoxing University

\section{Yubin Zou}

The Central Hospital Affiliated to Shaoxing University

Jiawei Lin

The Central Hospital Affiliated to Shaoxing University

\section{Bin Fang}

The Central Hospital Affiliated to Shaoxing University

Bing Wu ( $\nabla$ wubing@usx.edu.cn )

The Central Hospital Affiliated to Shaoxing University

\section{Research Article}

Keywords: miR-331-3p, OS, SOCS1, JAK2, STAT3

Posted Date: December 30th, 2021

DOI: https://doi.org/10.21203/rs.3.rs-1190352/v1

License: (c) (i) This work is licensed under a Creative Commons Attribution 4.0 International License. Read Full License 


\section{Abstract}

Background: MicroRNAs (miRNA) are regulatory small noncoding RNAs, which play a key role in many cancers. It has been found that miR-331-3p is involved in the development and progression of various cancers, but there are few reports in osteosarcoma.

Methods: The public GEO database was used to analyze the survival difference of miR-331-3p in OS organizations. The level of cell proliferation assay was assessed by CCK-8 and colony formation. Tanswell and Wound-healing detect the transfer and invasion ability of miR-331-3p in OS. TargetScan, miRDBmiR, TarBase, and dual luciferase reporter gene assays were used to determine SOCS1 as a targeted regulator. Western blot and immunohistochemistry were used to detect the expression of protein levels. A mouse model of subcutaneously transplantable tumors is used to evaluate the proliferation of OS in vivo.

Results: The low expression of miR-331-3p is negatively correlated with the overall survival of OS patients. Overexpression of miR-331-3p significantly inhibited cell proliferation, metastasis and invasion. miR-331-3p affects the occurrence and development of osteosarcoma by targeting the SOCS1/JAK2/STAT3 signaling pathway.

Conclusion: miR-331-3p reduces the expression of SOCS1 by combining with its 3 'UTR, thereby activating the JAK2/STAT3 signaling pathway to regulate the progression of OS.

\section{Background}

Osteosarcoma (OS) is one of the most common malignant bone tumors in children and adolescents [1]. The treatment for OS mainly comprises surgical resection, systemic chemotherapy, targeted radiation therapy, immunotherapy, gene therapy, and stem cell therapy [2,3]. Although surgical resection and neoadjuvant chemotherapy have reduced the mortality rates in surgical patients, the 5-year survival rate is still very low [4]. OS cell proliferation, migration, invasion, apoptosis, autophagy, epithelialmesenchymal transition, and other pathophysiological processes are closely related to the development of OS $[5,6]$. Unfortunately, the molecular mechanism of OS development has not yet been fully elucidated. Therefore, it is important to identify new diagnostic biomarkers and therapeutic targets.

MicroRNAs (miRNAs) are highly conserved endogenous non-coding RNAs with a length of approximately 19-25 nucleotides [7]. miRNAs are involved in many metabolic processes, including cell proliferation, differentiation, and apoptosis [8]. Many studies have confirmed that miRNA dysregulation is one of the causes of many cancers. miRNA mimics and miRNA-targeting molecules (antimiR) appear to be superior prospects for preclinical development [9]. For example, mimics of the tumor suppressor, miRNA miR-34, have entered phase I clinical trials for cancer treatment, and antimiRs targeting miR-122 have entered phase II trials for hepatitis treatment $[10,11]$. Interestingly, an increasing body of evidence has demonstrated the tumor suppressive potential of miR-331-3p in multiple cancers [12]. However, the functional role of miR-331-3p in OS remains unclear. 
Suppressor of cytokine signaling 1 (SOCS1) is a member of the SOCS family. It plays a vital role in regulating the immune system and in solving the inflammatory cascade $[13,14]$. SOCS1 is also considered to be a tumor suppressor in many cancers and may act as a tumor suppressor or promoter in a manner that depends on the cell environment [15]. We aimed to determine the expression profile of miR331-3p in osteosarcoma tissues using biological information and quantitative real-time polymerase chain reaction, and assess the effect of miR-331-3p on the growth of OS cells in vitro. We outline the potential mechanism of SOCS1 as a tumor suppressor and discuss new evidence of SOCS1 activity as an oncogene.

In this study, we explored the potential function of miR-331-3p in OS. Our results indicate that miR-331-3p can affect cell proliferation, migration and invasion ability in OS. At the same time, miR-331-3p activates JAK2/STAT3 phosphorylation level by inhibiting SOCS1. This indicates that miR-331-3p may be a target for the diagnosis and treatment of osteosarcoma.

\section{Materials And Methods}

\section{miRNA expression data analysis}

Gene array expression profile data GSE39040, including 65 OS tissues and 65 paired non-cancerous samples, were downloaded from the Gene Expression Omnibus database (GEO) [11]. All GEO data were analyzed in the $R$ statistical environment. The expression values of miR-331-3p from GEO data were analyzed using the online tool, ENCORI.

\section{Cell culture and transfection}

The human OS cell lines 143b, Hos, Saos-2, U-2OS and hFOB1.19 were obtained from the Cell Bank of the Chinese Academy of Sciences (Shanghai, China). HEK-293T cells were kindly provided by Sir Run Run Shaw Hospital, Zhejiang University School of Medicine \& Key Laboratory of Musculoskeletal System Degeneration and Regeneration Translational Research of Zhejiang Province. All cells were maintained in Dulbecco's Modified Eagle's Medium (Hyclone, LU, USA) supplemented with $10 \%$ fetal bovine serum (Gibco, NY, USA). The cells were incubated at $37^{\circ} \mathrm{C}$ in an atmosphere of $5 \% \mathrm{CO}_{2}$.

miR-331-3p inhibitor or miR-331-3p mimics were transfected into 143b and Hos cells separately. Cell transfection was performed using Lipofectamine 3000 (Invitrogen, Carlsbad, CA, USA) according to the manufacturer's instructions. After transfection for $48 \mathrm{~h}$, cells were harvested for further analysis.

\section{RNA extraction, reverse transcription, and quantitative real-time PCR}

Total RNA was extracted from OS cells using an Ultrapure RNA Purification Kit (Takara Bio, Shiga, Japan). Reverse transcription was performed using the Kr116 FastKing cDNA Synthesis Kit (TIANGEN, Beijing, China) according to the manufacturer's instructions. Quantitative real-time PCR (qRT-PCR) was performed in triplicate on a QuantStudio ${ }^{\mathrm{TM}} 3$ Real-Time PCR System using SuperReal PreMix Plus 
((TIANGEN, Beijing, China). miRNA quantification was determined by using Bulge-loop RT primer (RiboBio Co.LtdهGuangdong $\triangle$ China). Data obtained from qPCR were analyzed using GraphPad prism. Cycle threshold (Ct) values were calculated, and the relative mRNA levels of the target genes were analyzed using the $2-\triangle \Delta C T$ method. The primer sequence is as follows:

\begin{tabular}{lll} 
The target & Forward $\left(\mathbf{5}^{\prime}-\mathbf{3}^{\prime}\right)$ & Reverse $\mathbf{( 5 ^ { \prime } - \mathbf { 3 } ^ { \prime } )}$ \\
\hline SOCS1 & CCAGGTGGCAGCCGACAATG & CGAGGAGGAGGAAGAGGAGGAGA \\
\hline GAPDH & TGGTTGAGCACAGGGTACTT & CCAAGGAGTAAGACCCCTGG
\end{tabular}

\section{CCK-8 assay}

Transfected $143 \mathrm{~b}$ and Hos cells $\left(2 \times 10^{3}\right.$ cells) were cultured in a 96-well culture plate with $100 \mu \mathrm{L}$ of culture medium per well. $10 \mu \mathrm{L}$ of CCK-8 reagent (GLPBIO,CA囚USA) was added to each well at $0,24,48$, and $72 \mathrm{~h}$ post-treatment, followed by incubation for $3 \mathrm{~h}$. The plate was then shaken on a microplate reader, zero adjustment was performed using a blank hole, and absorbance was measured at $450 \mathrm{~nm}$ (optical density).

\section{Wound-healing assay}

Transfected 143b and Hos cells were cultured in serum-free medium for $24 \mathrm{~h}$. A $200-\mu \mathrm{L}$ pipette tip was used perpendicular to the 6-well plate to make scratches. Photographs were taken at $0 \mathrm{~h}$ and $24 \mathrm{~h}$ and analyzed with Image $\mathrm{J}$ software (National Institute of Health, Bethesda, MD, USA). The cell migration rate indicated the migration and movement abilities of each group of cells. The greater the migration rate, the greater the migration and movement ability.

\section{Cell migration and invasion assays}

Transfected 143b and Hos cells were cultured in serum-free medium for $24 \mathrm{~h}$ prior to migration and invasion assays. Cells $\left(0.2 \times 10^{5}\right)$ in $0.2 \mathrm{ml}$ of serum-free medium were seeded in the upper chamber containing $40 \mu \mathrm{L}$ of $1 \mathrm{mg} / \mathrm{ml}$ matrigel (BD Biosciences, MA, USA), and $0.6 \mathrm{ml}$ of complete medium containing $10 \%$ FBS was added to the lower chamber. After $48 \mathrm{~h}$ of incubation, the cells at the top of the membrane were removed with a cotton swab. The cells that migrated to the bottom well were fixed in methanol for $20 \mathrm{~min}$ and stained with $0.1 \%$ crystal violet solution. The number of invading cells was counted in three randomly selected optical microscope fields.

\section{Colony formation assay}

Transfected $143 \mathrm{~b}$ and Hos cells were plated into 6-well plates and cultured for 10 days at $37^{\circ} \mathrm{C}$. Colonies were then fixed with $4 \%$ paraformaldehyde for 20 min and stained with $0.1 \%$ crystal violet. The colony formation numbers ( $>50$ cells) were counted under a microscope, and the experiment was repeated three times over. 


\section{Dual-luciferase reporter assay}

Dual-luciferase reporter plasmids were purchased from Hanbio (Shanghai, China). HEK-293T cells were cultured in 6-well plates at a density of $3 \times 10^{4} \mathrm{cells} /$ well before transfection. Cells were then cotransfected with plasmid mixtures containing the RL reporter and FL reporter with or without the mir-3313p 3-UTR (500 ng) and SOCS13'-UTR or negative control (NC) (10 nM final concentration) using Lipofectamine RNAiMAX (Invitrogen囚AL区USA). Luciferase activity was measured using a Dual Luciferase Reporter Gene Assay Kit (Beyotime, Shanghai, China) after 48 h. For comparison, FLUC activity was normalized to RLUC activity to determine the ratio. The luciferase activity ratio of the miR-331-3p mimic group to the NC group was calculated and expressed as the fold-change.

\section{Western blot analysis}

Cells were harvested, washed, and lysed in lysis buffer (P0013, Beyotime, China) supplemented with a protease/phosphatase inhibitor cocktail. Proteins were separated using SDS-PAGE and transferred to poly vinylidene fluoride membranes (BioRad, AL, USA). The membrane was blocked overnight with $5 \%$ skim milk in Tris-buffered saline containing $0.5 \%$ Tween-20 and then incubated with primary antibodies against SOCS1 (Immunoway, USA), JAK2(Immunoway, USA), p-JAK2(Immunoway, USA), STAT3(Immunoway, USA), p-STAT3(Immunoway, USA) and $\beta$-actin (Immunoway, USA) at $4^{\circ} \mathrm{C}$. The membranes were then incubated with an HRP secondary antibodies (Dawen, HangZhou凶China). Proteins were detected using an enhanced chemiluminescence kit (Beyotime, Shanghai, China) according to the manufacturer's instructions. An anti- $\beta$-actin antibody was used to detect uniform loading. Each experiment was performed in triplicate.

\section{Subcutaneous xenograft tumor models}

Approximately $5 \times 10^{6} 143 \mathrm{~b}$ cells were injected subcutaneously into nude mice (female, 4 weeks old) ( $\mathrm{n}=6$ per group) by randomization. The width and length of the tumor was measured for 4 consecutive weeks. The tumor volume was calculated as follows: volume $\left(\mathrm{mm}^{3}\right)=\left(\right.$ length $\times$ width $\left.^{2}\right) / 2$. Mice were sacrificed 4 weeks after injection, and the tumors were collected and weighed. A part of the tumor was extracted for RNA and protein analysis, and the remaining tissue was fixed in $4 \%$ paraformaldehyde for further use.

\section{Plasmid construction and stable transfection}

Stable transfection small interfering RNAs (siRNAs) were obtained from RiboBio (Guangzhou, China) and transfected into cells using Lipofectamine iMax (Invitrogen囚AL囚USA) following the manufacturer's instructions. The human lentivirus miR-331-3p sponge and the SOCS1-overexpressing lentiviral plasmid were purchased from HanBio. miRNA mimics and inhibitors were purchased from GenePharma (Shanghai, China). Transfection efficiency was verified using qRT-PCR.

\section{Immunohistochemistry (IHC)}


Primary antibody against SOCS1 (Immunoway, USA) was used and incubated with the cells overnight at $4{ }^{\circ} \mathrm{C}$. Thereafter, the cells were washed in phosphate-buffered saline (PBS), followed by incubation with secondary antibodies (Proteintech IL, USA) for $1 \mathrm{~h}$. Finally, the cells were washed in PBS, and immunofluorescence images were captured using an inverted microscope (Nikon, Tokyo, Japan).

\section{Statistical analyses}

All statistical analyses were performed using GraphPad Prism 6 software. Statistical significance was defined as a two-sided P-value $<0.05$. Experiments were replicated at least three times, and data are shown as mean \pm standard deviation (SD) in bars.

\section{Results}

\section{The expression of miR-331-3p was decreased in OS}

We analyzed the expression level of miR-331-3p in osteosarcoma through bioinformatics. The analysis results showed that low expression of miR-331-3p was negatively associated with overall survival (days) in patients with OS (Figure 1A). Secondly, we detected the expression of miR-331-3p in U-20S, HOS, Saos$2,143 \mathrm{~b}$, and hFOB1.19 by qRT-PCR. The results indicated that miR-331-3p is down-regulated in OS cell lines compared with normal cells (Figure 1B). These data indicate that miR-331-3p is closely related to the occurrence and development of OS.

\section{MiR-331-3p suppresses OS cell proliferation metastasis, and invasion in vitro}

The complex role of miR-331-3p as an oncogene or tumor suppressor has been described in several cancer types, but rarely in OS. Here, we evaluated the role of miR-331-3p in OS by transfecting 143b and Hos cells with miR-331-3p mimics or inhibitors. We detecting transfection efficiency using qRT-PCR (Figure 2A). Furthermore囚the CCK-8 detection and plate colony formation analysis showed that miR-331$3 p$ over-expression further inhibited cell proliferation compared with the NC group, while miR-331-3p inhibition resulted in increased cell proliferation (Figure 2B-C). In addition, wound healing and transwell assays confirmed that when miR-331-3p was overexpressed, the migration and invasion ability of OS cells decreased significantly, while miR-331-3p was inhibited, 143b and Hos showed an upward trend (Figure 2D-E). Overall, these data indicated that miR-331-3p can inhibit the migration, invasion, and proliferation of OS cells in vitro.

\section{SOCS1 is a direct target of miR-331-3p in OS}

Prediction of SOCS1 and NACC1 as possible targets of miR-331-3p was performed by analysis of the three databases, TargetScan, miRDB, and miRTarBase (Figure 3A). The results showed that SOCS1 had the greatest promotion effect on cell proliferation by qRT-PCR (Figure 3B); therefore, it was selected for further research. The results of the dual luciferase reporter assay verified the targeting relationship between miR-331-3p and SOCS1 (Figure 3C-D). Furthermore, qRT-PCR and western blot analysis revealed 
that SOCS1 expression was inversely regulated by miR-331-3p mimics or inhibitors in OS cells, suggesting that SOCS1 is a true miR-331-3p target (Figure 3E-F).

It has been reported that SOCS1 acts as an oncogene in various cancers, but its role in OS is unclear [15]. To determine whether the expression of endogenous SOCS1 is closely related to the pathogenesis of OS, $143 \mathrm{~b}$ and Hos cells were transfected with si- SOCS1 1 to evaluate their functions in vitro. Transfection efficiency was verified by qRT-PCR (Figure S1A). Wound healing and transwell assays showed that lower levels of SOCS1 inhibited OS cell metastasis and invasion (Figure S1B-C). On the other hand, compared with the control group, OS cells with down-regulated SOCS1 expression had a lower growth rate and reduced colony formation (Figure S1D). The CCK-8 results showed that lower levels of SOCS1 significantly inhibited cell proliferation (Figure S1E). In summary, our results indicate that over-expression of miR-331-3p may inhibit the progression of OS through SOCS1.

\section{miR-331-3p promotes OS progression via SOCS1}

The SOCS1 over-expression plasmid was constructed and transfected into 143b and Hos cells with stable over-expression of miR-331-3p to further investigate whether miR-331-3p affects OS progression by targeting SOCS1. The increased cell viability (Figure 4A) and proliferation (Figure 4B) capabilities of OS cells by miR-331-3p overexpression were all abolished by SOCS1 overexpression. In addition, it was found that high expression of SOCS1 can rescue the down-regulation of miR-331-3p-deficient cells through cell migration and transwell experiments (Figure 4C-D). Protein and gene levels also verified the above phenomenon (Figure 4E-F). Taken together, these findings indicate that miR-331-3p is involved in OS progression by targeting SOCS1.

\section{miR-331-3p regulates the biological functions of OS through the SOCS1/JAK2/STAT3 pathway}

Then, we further explore the underlying molecular mechanisms of miR-331-3p. According to the reports, the exosomal miR-221-3p derived from M2-TAMs aggravates OS progression via modulating the SOCS3/JAK2/STAT3 axis. We found that miRNA-331-3p activates JAK2/STAT3 phosphorylation level by inhibiting SOCS1 (Figure 5A-B). These results suggested that miRNA-331-3p may act through the SOCS1/JAK2/STAT3 pathway.

\section{SOCS1 acts as a sponge for miR-331-3p to promote tumorigenesis in vivo}

An over-expression/silencing SOCS1 lentiviral plasmid was constructed and transfected into OS cells. The stabilized cells were used to construct a mouse orthotopic xenograft model to determine whether miR-331-3p and SOCS1 also function in vivo. The tumor volume of the miR-331-3p over-expression group was significantly smaller than that of the control group, while the co-transfection group showed a reversed trend (Figure 6A). The tumor weights of the three groups also showed the same difference (Figure 6B). The up-regulated expression of miR-331-3p also suppressed tumor volume in subcutaneous xenograft models (Figure 6C). In addition, miR-331-3p mimics significantly reduced SOCS1 mRNA expression levels, and the co-transfection group showed a reversed trend (Figure 6D). Western blot results 
indicate that miR-331-3p affects the occurrence and development of osteosarcoma through the SOCS1/JAK2/STAT3 pathway (Figure 6E). The results of IHC showed that the average immunopositive area of SOCS1 decreased under the influence of miR-331-3p, and SOCS1 offset this change again (Figure $6 \mathrm{~F})$. These results indicate that miR-331-3p may participate in processes such as OS growth and metastasis through SOCS1.

\section{Discussion}

miRNAs are important regulators of gene expression and are involved in a variety of cellular physiological processes, such as cell growth, movement, and differentiation [16]. Nowadays, more and more evidence has confirmed that miRNAs play a key role in cancer malignancies by acting as tumor suppressor genes or oncogenes. Therefore, miRNA can be used as an important indicator of prognosis and treatment [17]. miR-331-3p is a newly discovered miRNA that has been confirmed as a tumor suppressor, and it is usually down-regulated in different cancers. For example, miR-331-3p is significantly down-regulated in triplenegative breast cancer (TNBC) tissues and cell lines. One study reported that over-expression of miR-331$3 p$ inhibits the increase in proliferation and apoptosis of TNBC cells [18]. The expression of miRNA-331$3 p$ in clinical specimens and cells of nasopharyngeal carcinoma was also significantly reduced, while the over-expression of miRNA-331-3p inhibited cell proliferation and invasion. In addition, over-expression of miRNA-331-3p reduced the expression of the target gene elF4B, thereby inhibiting phosphorylation of phosphoinositide 3-kinase and serine/threonine kinase [19]. However, little is known about the biological functions of miR-331-3p in OS.

In this study, we used bioinformatics to analyze GEO and TCGA data and found that low level of miR-331$3 p$ is closely related to overall survival rate and is an independent prognostic factor affecting the overall survival rate of patients. The qRT-PCR results showed that the expression of miR-331-3p was significantly down-regulated in the OS cell line, confirming this conclusion. Functional tests showed that the overexpression of miR-331-3p inhibited the metastasis and invasion ability of OS cells in vitro and reduced cell viability and proliferation ability, and knockdown of miR-331-3p promotes cell migration and invasion. Our results indicated that miR-331-3p is an inhibitory miRNA in OS cells. This is consistent with previous studies of other cancers.

SOCS1, also known as STAT-induced STAT inhibitor (SSI) or JAK binding protein (JAB), is a member of the SOCS family [20]. SOCS1 negatively regulates cytokines by activating the JAK-STAT signaling pathway[21]. According to literature reports, $\mathrm{m} 6 \mathrm{~A}$ mRNA controls $\mathrm{T}$ cell methylation by targeting the IL7/STAT5/SOCS pathway[22]. HuoXueTongFu Formula Alleviates Intraperitoneal Adhesion by Regulating Macrophage Polarization and the SOCS/JAK2/STAT/PPAR- $y$ Signalling Pathway[23]. Recent studies have shown that SOCS1 can play a role in cancer by regulating oncogenic signal transduction pathways. Zhang Mao et al. found that SOCS5 can inhibit HCC cell migration and invasion in vitro by activating $\mathrm{PI3K} / \mathrm{Akt} / \mathrm{mTOR}$-mediated autophagy[24]. Here, we through the analysis of the three databasesTargetScan, miRDB, and miRTarBase-we speculate that SOCS1 is the target gene of miR-331-3p. The dual luciferase results confirmed that SOCS1 was the target effector of miR-331-3p. The rescue 
experiment once again verified that miR-331-3p inhibits the occurrence of OS cells and tumors by regulating SOCS1. Next, we continue to explore possible miR-331-3p/SOCS1 regulatory pathways. Through western blot, we found that miR-331-3p activates the JAK2/STAT3 pathway by inhibiting SOCS1, thereby affecting the progress of OS (Figure 7A).

\section{Conclusion}

In conclusion, our results confirm that miR-331-3p inhibits the proliferation, metastasis and invasion of osteosarcoma cells. Further mechanism studies found that miR-331-3p participates in targeting SOCS1 and up-regulates JAK2/STAT3 to affect the progression of OS. The molecular mechanism explored in this study may provide a new theoretical basis for the pathogenesis and treatment of osteosarcoma.

\section{Abbreviations}

osteosarcoma (OS); microRNAs (miRNAs); suppressor of cytokine signaling 1 (SOCS1); negative control (NC); small interfering RNAs (siRNAs); immunohistochemistry (IHC); phosphate-buffered saline (PBS); triple-negative breast cancer (TNBC); fetal bovine serum (FBS).

\section{Declarations}

\section{Ethics approval and consent to participate}

This work is carried out in accordance with the principles contained in the Declaration of Helsinki.

Research involving human participants was reviewed and approved by the Experimental Animal Welfare Ethics Committee of Zhejiang Provincial Laboratory Animal Center.

\section{Consent for publication}

All authors involved in the authorship are consent for publication in the current form.

\section{Availability of data and materials}

The datasets used and/or analysed during the present are available from the corresponding author on reasonable request.

\section{Competing interests}

The authors report no conflicts in this work.

\section{Funding}

This work was supported by the Medical and Health Science and Technology Plan Project of Zhejiang Province (grant number 2020KY990). 
Authors' contributions

WB and FB designed experiments. ZD and DQ conducted molecular research and conducted experiments. CSF, CYD, YJ, ZYB and LJW contributed to data analysis and interpretation. ZD drafted the manuscript. All authors approved the final manuscript.

\section{Acknowledgements}

The authors would like to thank the teachers in Zhejiang Provincial Laboratory Animal Center, for their kindly help in mastering the skills of animal experiments. In addition, the authors thank the teachers in the Sir Run Run Shaw Hospital for their guidance in the experiment.

\section{Author details}

${ }^{a}$ Central Laboratory, The Central Hospital Affiliated to Shaoxing University, Shaoxing, 312030, China. ${ }^{\text {bDepartment }}$ of Spine Surgery, The Central Hospital Affiliated to Shaoxing University, Shaoxing, 312030, China.

\section{References}

1. Wittig JC, Bickels J, Priebat D, Jelinek J, Kellar-Graney K, Shmookler B, Malawer MM: Osteosarcoma: a multidisciplinary approach to diagnosis and treatment. Am Fam Physician 2002, 65(6):1123-1132.

2. ElKordy MA, ElBaradie TS, EISebai HI, KhairAlla SM, Amin AAE: Osteosarcoma of the jaw: Challenges in the diagnosis and treatment. J Egypt Natl Canc Inst 2018, 30(1):7-11.

3. Wang J, Liu S, Shi J, Li J, Wang S, Liu H, Zhao S, Duan K, Pan X, Yi Z: The Role of miRNA in the Diagnosis, Prognosis, and Treatment of Osteosarcoma. Cancer Biother Radiopharm 2019, 34(10):605613.

4. Reed DR, Hingorani P, Anderson PM: Relapsed Osteosarcoma Trial Concepts to Match the Complexity of the Disease. Adv Exp Med Biol 2020, 1257:85-94.

5. Cersosimo F, Lonardi S, Bernardini G, Telfer B, Mandelli GE, Santucci A, Vermi W, Giurisato E: TumorAssociated Macrophages in Osteosarcoma: From Mechanisms to Therapy. Int J Mol Sci 2020, 21(15).

6. Camuzard O, Santucci-Darmanin S, Carle GF, Pierrefite-Carle V: Role of autophagy in osteosarcoma. $J$ Bone Oncol 2019, 16:100235.

7. Lu TX, Rothenberg ME: MicroRNA. J Allergy Clin Immunol 2018, 141(4):1202-1207.

8. Giza DE, Calin GA: microRNA and Chronic Lymphocytic Leukemia. Adv Exp Med Bio/ 2015, 889:23-40. 
9. lorio MV, Croce CM: MicroRNA dysregulation in cancer: diagnostics, monitoring and therapeutics. A comprehensive review. EMBO Mol Med 2017, 9(6):852.

10. Zhang L, Liao Y, Tang L: MicroRNA-34 family: a potential tumor suppressor and therapeutic candidate in cancer. J Exp Clin Cancer Res 2019, 38(1):53.

11. Rupaimoole R, Slack FJ: MicroRNA therapeutics: towards a new era for the management of cancer and other diseases. Nat Rev Drug Discov 2017, 16(3):203-222.

12. Chen T, Ma L, Cui J, Geng J, Zeng Y, Chen W: [Construction of miR-331-3p overexpression vector and its effect on cell proliferation]. Sheng Wu Gong Cheng Xue Bao 2019, 35(5):892-900.

13. He C, Yu CR, Mattapallil MJ, Sun L, Larkin lii J, Egwuagu CE: SOCS1 Mimetic Peptide Suppresses Chronic Intraocular Inflammatory Disease (Uveitis). Mediators Inflamm 2016, 2016:2939370.

14. llangumaran S, Bobbala D, Ramanathan S: SOCS1: Regulator of T Cells in Autoimmunity and Cancer. Curr Top Microbiol Immunol 2017, 410:159-189.

15. Beaurivage C, Champagne A, Tobelaim WS, Pomerleau V, Menendez A, Saucier C: SOCS1 in cancer: An oncogene and a tumor suppressor. Cytokine 2016, 82:87-94.

16. Saliminejad K, Khorram Khorshid HR, Soleymani Fard S, Ghaffari SH: An overview of microRNAs: Biology, functions, therapeutics, and analysis methods. J Cell Physiol 2019, 234(5):5451-5465.

17. Shah V, Shah J: Recent trends in targeting miRNAs for cancer therapy. J Pharm Pharmaco/ 2020, 72(12):1732-1749.

18. Zhao M, Zhang M, Tao Z, Cao J, Wang L, Hu X: miR-331-3p Suppresses Cell Proliferation in TNBC Cells by Downregulating NRP2. Technol Cancer Res Treat 2020, 19:1533033820905824.

19. Xuefang Z, Ruinian Z, Liji J, Chun Z, Qiaolan Z, Jun J, Yuming C, Junrong H: miR-331-3p Inhibits Proliferation and Promotes Apoptosis of Nasopharyngeal Carcinoma Cells by Targeting elf4B-PI3K-AKT Pathway. Technol Cancer Res Treat 2020, 19:1533033819892251.

20. Sharma J, Larkin J, 3rd: Therapeutic Implication of SocS1 Modulation in the Treatment of Autoimmunity and Cancer. Front Pharmacol 2019, 10:324.

21. Durham GA, Williams JJL, Nasim MT, Palmer TM: Targeting SOCS Proteins to Control JAK-STAT Signalling in Disease. Trends Pharmacol Sci 2019, 40(5):298-308.

22. Li HB, Tong J, Zhu S, Batista PJ, Duffy EE, Zhao J, Bailis W, Cao G, Kroehling L, Chen Y et al: m(6)A mRNA methylation controls $T$ cell homeostasis by targeting the IL-7/STAT5/SOCS pathways. Nature 2017, 548(7667):338-342. 
23. Zhao M, Bian YY, Yang LL, Chen YQ, Wang YJ, Ma YT, Pei YQ, Li WL, Zeng L: HuoXueTongFu Formula Alleviates Intraperitoneal Adhesion by Regulating Macrophage Polarization and the SOCS/JAK2/STAT/PPAR-gamma Signalling Pathway. Mediators Inflamm 2019, 2019:1769374.

24. Zhang M, Liu S, Chua MS, Li H, Luo D, Wang S, Zhang S, Han B, Sun C: SocS5 inhibition induces autophagy to impair metastasis in hepatocellular carcinoma cells via the PI3K/Akt/mTOR pathway. Cell Death Dis 2019, 10(8):612.

\section{Figures}

A

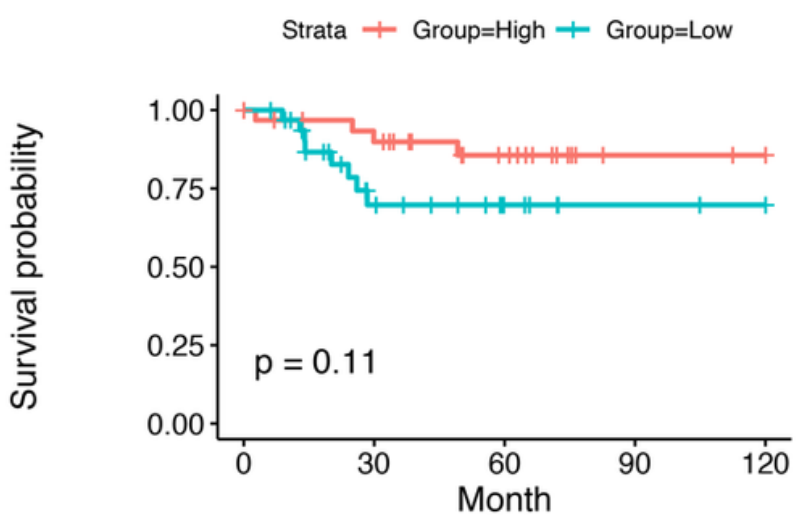

Number at risk

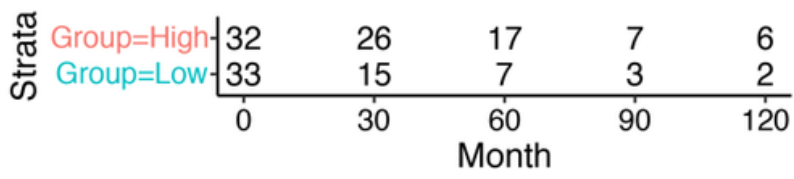

B

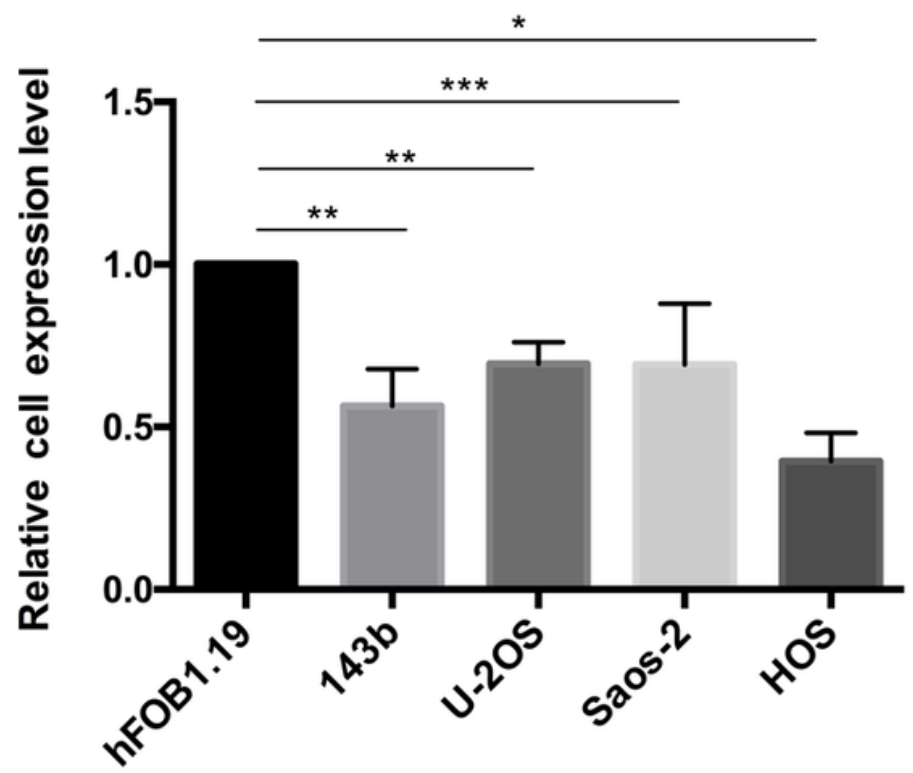

Figure 1

The expression of miR-331-3p decreases in OS. (A) The survival curve of the differential expression of miR-331-3p in OS. (B) qRT-PCR detects the expression level of miR-331-3p in OS cell lines. The results are presented as mean \pm standard deviation (SD) from three independent experiments. ${ }^{*} P<0.01$.

Figure 2

miR-331-3p can inhibit the proliferation, migration and invasion of OS in vitro. (A)143b and Hos are transfected with miR-331-3p mimics or inhibitors, and then cell biological functions are evaluated. (A) qRT-PCR detects the transfection efficiency of miR-331-3p. (B) The cell viability of 143b and Hos after the detection of miR-331-3p mimics/inhibitors by CCK-8. (C) Colony formation analysis of changes in OS cell proliferation ability. (D) The transwell experiment detects the invasion level of OS cells. (E) The migration ability of OS cells is analyzed by wounding experiment. The results are shown as the mean \pm standard deviation (SD) from three independent experiments. ${ }^{* *} \mathrm{P}<0.01$. 


\section{Figure 3}

SOCS1 is a direct target of miR-331-3p and is considered an oncogene in OS. (A)Screening target genes through the intersection of three databases (TargetScan, miRDB, and miRTarBase). (B) qRT-PCR is used to evaluate the expression level of potential target genes in OS cells. (C) Schematic diagram of the binding sequence between miR-331-3p and SOCS1. (D) HEK-293T cells are transfected with miR-331-3p mimic or negative control (NC) and wild-type or mutant SOCS13'-UTR, and subjected to luciferase assay. (E) qRT-PCR is used to evaluate the mRNA level of SOCS1 in OS cells transfected with miR-331-3p mimics/inhibitors. (F) Western blotting is used to evaluate the protein expression of SOCS1 in OS cells transfected with miR-331-3p mimics/inhibitors. The results are shown as the mean \pm standard deviation (SD) from three independent experiments. ${ }^{*} \mathrm{P}<0.01$. 
143b

A

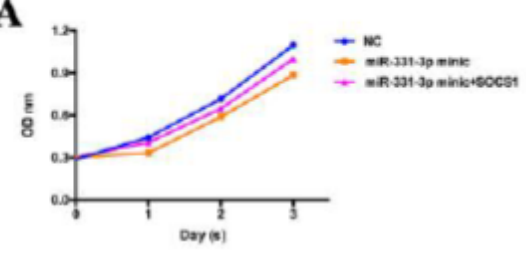

Hos

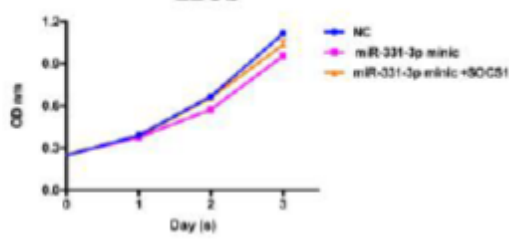

B

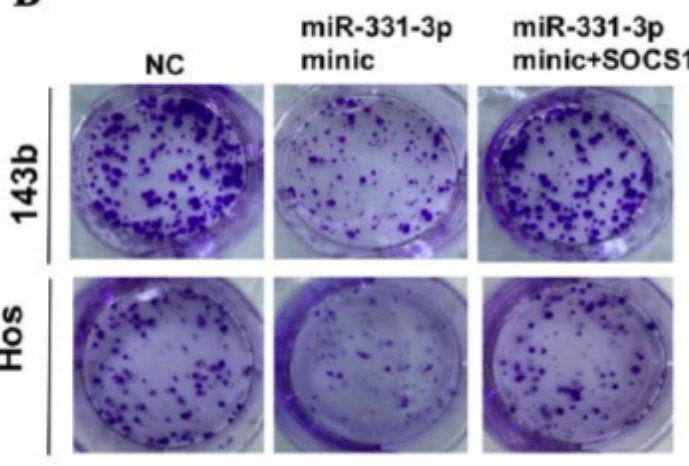

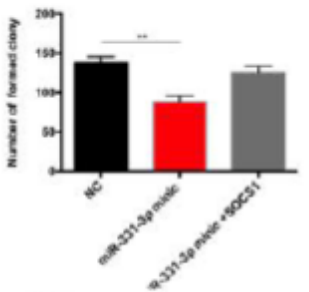

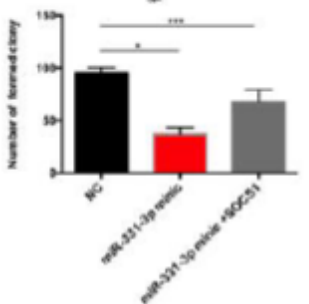

C

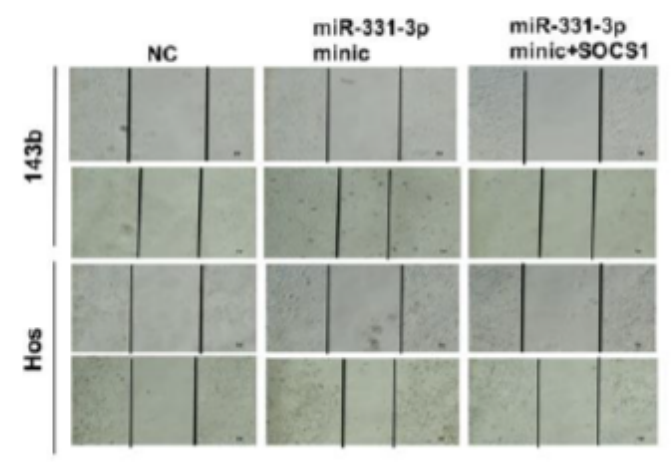

E

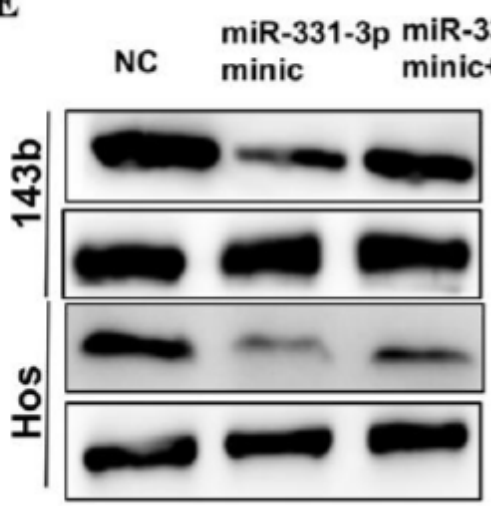

D

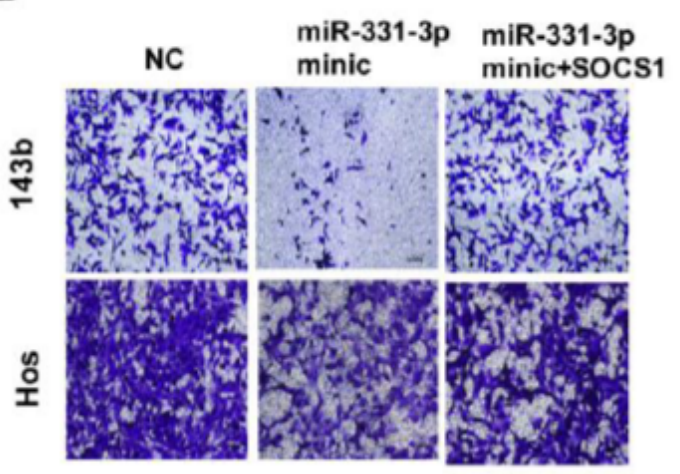

F

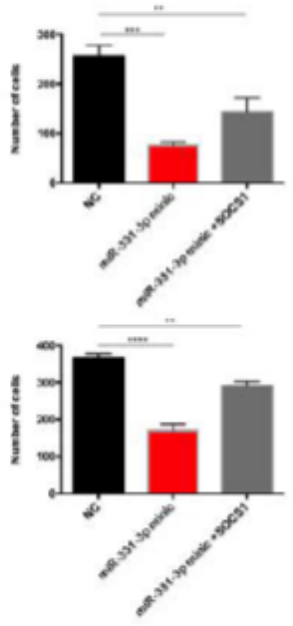

143b

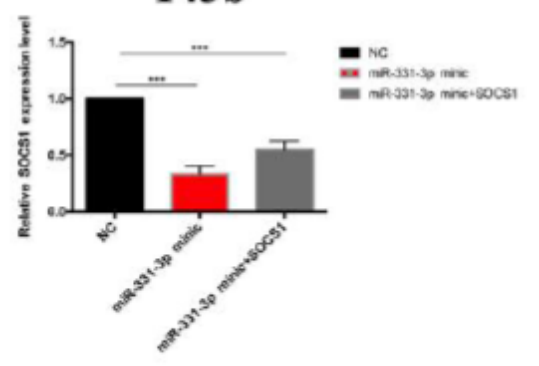

Hos

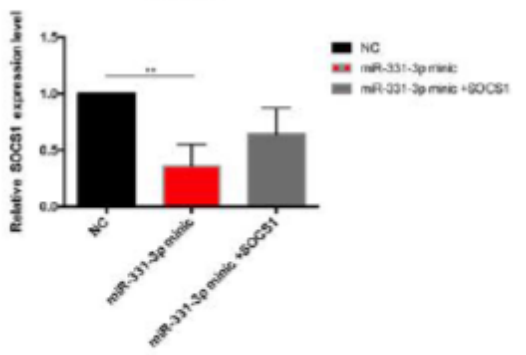

Figure 4

SOCS1 regulates the tumor suppressor function of miR-331-3p in OS cells in vitro. (A)CCK-8 assay shows that miR-331-3p over-expression inhibits the effect of SOCS1 silencing on cell growth. (B) Colony formation analysis of changes in OS cell proliferation ability. (C) Wound repair experiments show the effect of SOCS1 over-expression on migration ability. (D) The invasion ability of cells after co-transfection with miR-331-3p and SOCS1 is determined by the invasion test. (E) Western blot is used to detect the protein expression level of SOCS1 in OS cells after miR-331-3p alone transfection and miR-331-3p and SOCS1 co-transfection. (F) Detection of SOCS1 mRNA expression level in OS cells after miR-331-3p alone 
transfection and miR-331-3p and SOCS1 co-transfection by quantitative real-time polymerase chain reaction (qRT-PCR). The results are shown as the mean \pm standard deviation (SD) from three independent experiments. ${ }^{*} \mathrm{P}<0.01$.
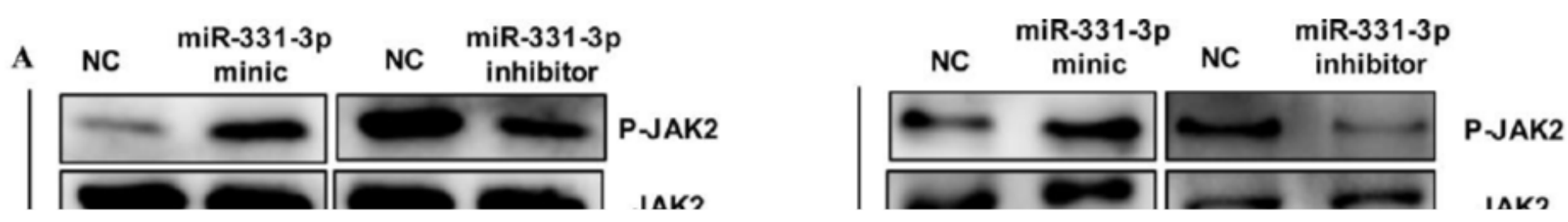

\section{Figure 5}

miR-331-3p inhibits SOCS1 leading to activation of the JAK2/STAT3 pathway. (A) OS cells transfected with miR-331-3p mimic/inhibitor. Protein expressions of JAK2, p-JAK2, STAT3, and p-STAT3 were examined by Western blot. (B) OS cells transfected with NC or miR-331-3p minic or cotransfected with miR-331-3p minic and the SOCS1 overexpression plasmid. Protein expressions of JAK2, p-JAK2, STAT3, and p-STAT3 were examined by Western blot. 


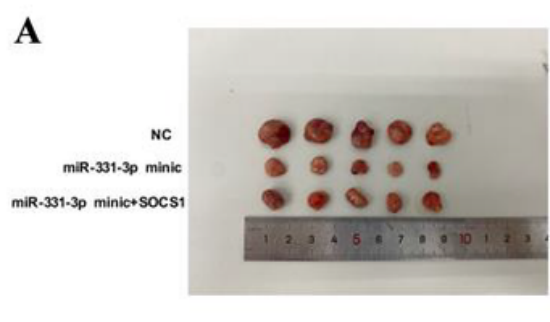

B

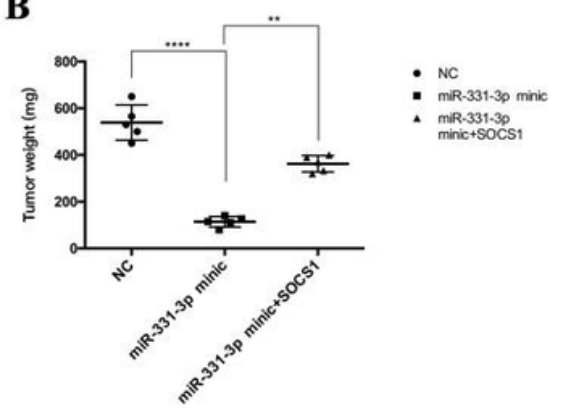

D

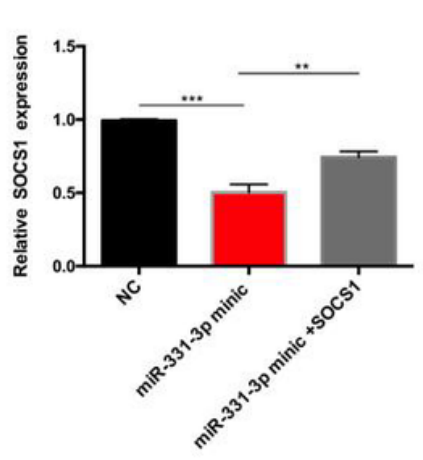

E

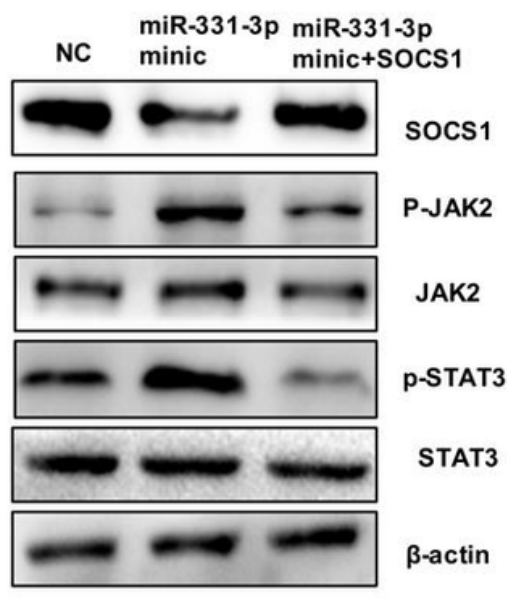

C

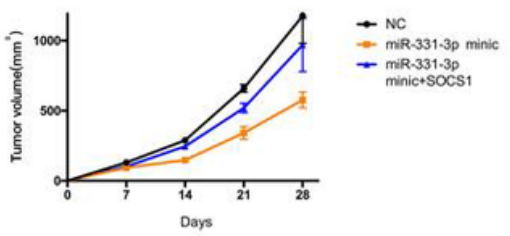

F

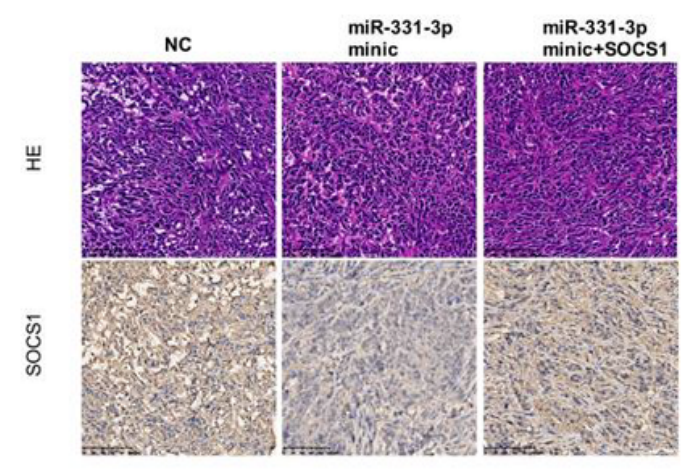

\section{Figure 6}

SOCS1 acts as a target of miR-331-3p to promote tumorigenesis in vivo. 143b cells are inoculated subcutaneously in female nude mice at a density of $5 \times 10^{6}$ cells. (A) After 4 weeks, the tumor is dissected and imaged. (B) Average tumor weight of mice. The data represents the mean \pm standard error of the mean (SEM) ( $n=5$ per group). (C) Tumor volume $\left(a b^{2} / 2\right)$ was recorded every seven days after mice were injected with stable OS cells. The data represents the mean \pm SEM ( $n=5$ per group). (D) qRT-PCR analysis of SOCS1 mRNA expression in xenograft mouse tumors. ( $n=5$ in each group). (E) Western blot analysis of SOCS1冈JAK2, p-JAK2, STAT3, and p-STAT3 protein expression levels in different groups of tumors. (F) HE and Immunohistochemistry staining shows the tumor structure and relative protein level of SOCS1 in tumors. The results are shown as the mean \pm standard deviation (SD) from three independent experiments. ** $P<0.01$. 


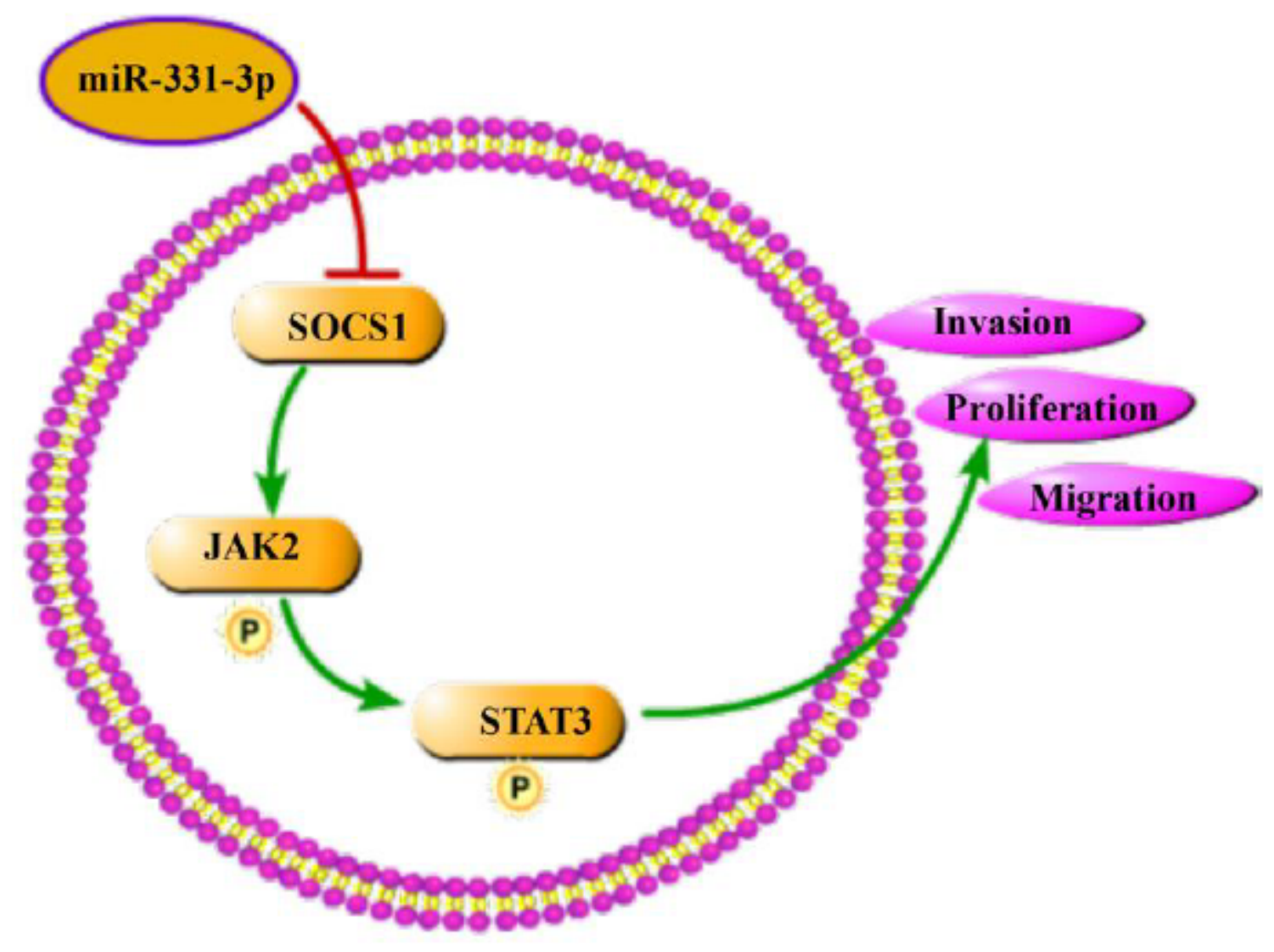

Figure 7

Graphical abstract. miR-331-3p targets the inhibition of SOCS1, thereby activating the phosphorylation level of JAK2/STAT3, and affecting the proliferation, migration and invasion of OS cells. The arrow indicates activation, and the T symbol indicates inhibition.

\section{Supplementary Files}

This is a list of supplementary files associated with this preprint. Click to download.

- Supplementarymaterial.docx 